\title{
The growth channel of massive galaxies
}

\author{
Ignacio Trujillo ${ }^{1,2}$ \\ ${ }^{1}$ Departamento de Astrofísica, Universidad de La Laguna, E-38205, La Laguna, Tenerife, Spain \\ ${ }^{2}$ Instituto de Astrofísica de Canarias, c/ Vía Láctea s/n, E-38205, La Laguna, Tenerife, Spain \\ email: trujillo@iac.es
}

\begin{abstract}
There is growing evidence suggesting that massive galaxies have growth both in mass and size mainly by the accretion of smaller satellites. This evolutionary path qualitative explains many different observations. However, there is still much work to do on trying to quantify whether this merging scenario is enough to explain the significant growth of massive galaxies since $\mathrm{z} \sim 2$. In this contribution, we show that both the number of satellite galaxies around massive galaxies at all redshifts, and the existence of massive relic galaxies in the nearby Universe are in quantitative agreement with this merging picture. Nonetheless, there is still open questions related to the properties of the stellar halos of present-day massive galaxies and the number density of massive relics at all redshifts that remain open.
\end{abstract}

\section{Introduction}

How did the enormous cosmic structural evolution of the most massive galaxies take place (Daddi et al. 2005; Trujillo et al. 2006)? There is a currently growing consensus that the size evolution (around a factor of 4 since $\mathrm{z} \sim 2$; Trujillo et al. 2007; Buitrago et al. 2008) was driven by a continuous accretion of satellites towards the main objects (e.g. Bezanson et al. 2009; Hopkins et al. 2009; Oser et al. 2010). However, although in-situ growth scenarios (Fan et al. 2008; Ragone-Figueroa \& Granato 2011) have been disfavored observationally (see a full discussion in e.g. Trujillo, Ferreras, \& de La Rosa 2011), exsitu models (i.e. merging scenarios Ciotti \& van Albada 2001; Khochfar \& Burkert 2006; Naab, Johansson, \& Ostriker 2009) lack a detailed quantitative confrontation with the observational data. What follows are two observational tests that merging scenarios have to observationally pass to be considered as a valid growth channel:

- Have we got enough number of mergers to produce the size increase?

- Where are the untouched massive relic galaxies in the nearby Universe?

We will expand on these questions in the following sections.

\section{Have we got enough number of mergers to produce the size increase?}

If the merging channel is the predominant path that massive galaxies have followed to reach their current sizes, at all redshifts there should be enough satellites surrounding the massive galaxies to produce, by accretion, the observed size evolution. There is an increasing number of papers exploring the frequency of satellites around the most massive galaxies at different cosmic epochs (e.g. Kaviraj et al. 2009; Bundy et al. 2009; MármolQueraltó et al. 2012; López-Sanjuan et al. 2012; Ruiz, Trujillo, \& Mármol-Queraltó 2014). The strategy followed in those works consists on counting the number of galaxies within a given radial distance and redshift interval to the targeted massive galaxies. This is illustrated in Fig. 1. The amount of satellites around massive galaxies have remained pretty much constant with cosmic time at least since $\mathrm{z} \sim 1.5$. This is quite independent of the mass ratio between the host and the satellite galaxy. This is shown in Fig. 2. 

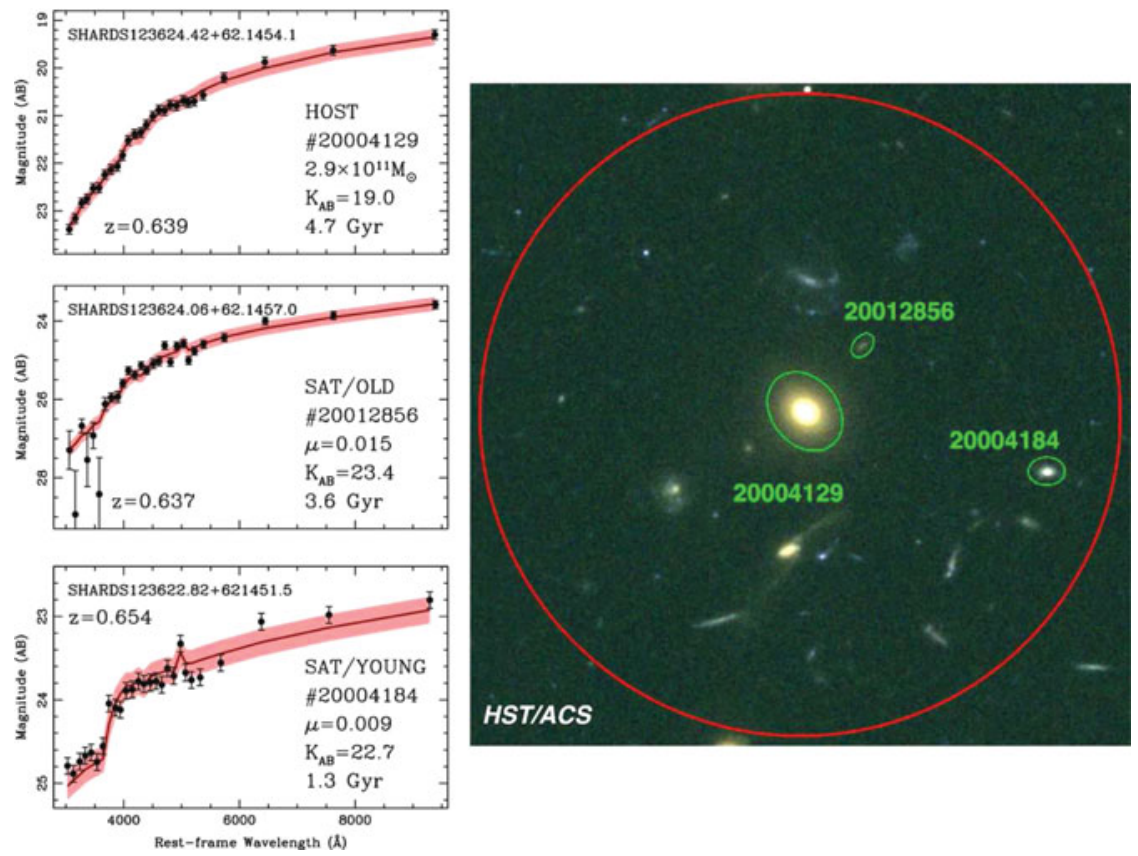

Figure 1. Illustration of how satellites are identified around massive galaxies (figure taken from Ferreras et al. 2014). The host is the massive galaxy SHARDS123624.42+621454 ( $\mathrm{z}=0.639$, $\mathrm{M}_{\star}=2.9 \times 10^{11} \mathrm{M}_{\odot}$ ). The $30 \times 30 \operatorname{arcsec}^{2}$ image (right) is an RGB colour composite from the GOODS-N HST/ACS archival data through the F606W, F775W, and F850LP passbands. The red circumference extends over the $100 \mathrm{~h}_{70}^{-1} \mathrm{kpc}$ search radius. On the left, the observed data points (black circles) are compared with the best-fitting models (red lines). Each galaxy is labeled by its SHARDS ID, $\mathrm{K}_{A B}$ apparent magnitude, best-fitting stellar age, and either stellar mass (host) or mass ratio (companion).

A detailed analysis of the mass contribution of each satellite to the whole system hostsatellites shows that, in case of infall of the satellites to the galaxy host, the largest contributor to the mass growth of the host would be those satellites with the largest masses (Ruiz, Trujillo, \& Mármol-Queraltó 2014; Ferreras et al. 2014). This is because there are not enough number of small satellites to balance the contribution of the most massive satellites.

In Fig. 3 we show what would be the average mass growth of the massive galaxies assuming two different infall timescale of the satellites to the host galaxies. The first one assumes that the infall time scale is independent of the mass of the satellite. The other one takes into account the dynamical friction (Jiang, Jing, \& Han 2014), and consequently, considers more likely that the most massive satellites have shorter infall time scales. Independently, of the exact details of the infall timescale, the average mass growth of the massive galaxies due to the infall of satellites is $\sim 8 \%$ per Gyr. This translates into a global mass growth since $\mathrm{z} \sim 1$ of approximately a factor of 2 .

According to the literature, the growth in size is connected to the growth in mass by simple scaling relationships: $\Delta \mathrm{R} \propto \Delta \mathrm{M}^{\alpha}$ with $1 \lesssim \alpha \lesssim 2$. Consequently, an increase in mass of $\sim 2$ will correspond to an increase in size of $\sim 2-4$. This is more than enough to explain the observed size evolution of the massive galaxies since $\mathrm{z} \sim 1$, which is around a factor of 2 (e.g. Trujillo et al. 2007). In this sense, the merger channel seems to have enough number of satellites around the massive galaxies to be able to explain the observed size growth without significant problems. 


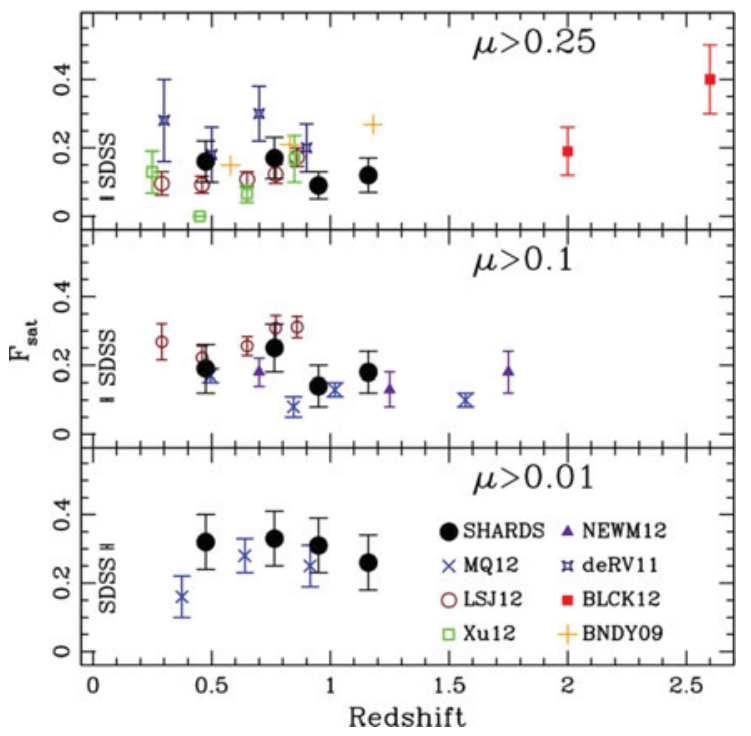

Figure 2. Redshift evolution of the fraction of close companions of massive galaxies $\left(M_{\star}>\right.$ $\left.10^{11} \mathrm{M}_{\odot}\right)$, for three choices of the stellar mass ratio between satellite and central $(\mu)$. The solid circles (Ferreras et al. 2014) are compared with recent results from the literature.

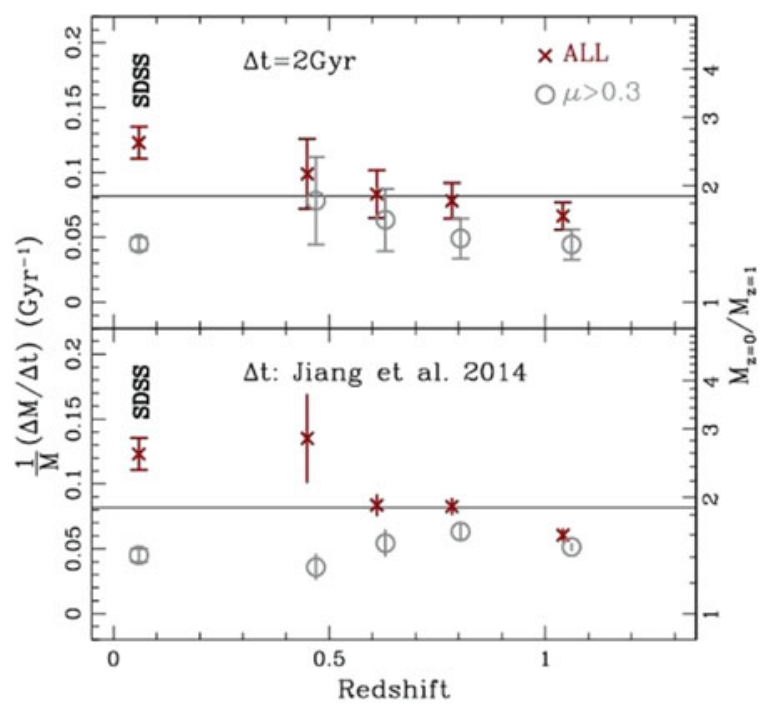

Figure 3. Top: growth rate of massive galaxies with respect to redshift for the simple case of a fixed $(\Delta t=2$ Gyr $)$ merging time-scale. The red crosses (grey circles) correspond to the contribution from the whole sample (only from major mergers, $\mu>0.3$ ). The axis on the right gives the expected stellar mass growth between $\mathrm{z}=1$ and 0 assuming a constant rate. For reference, the points on the left, labelled SDSS, correspond to a nearby sample. Bottom: same as above, although taking into account the dependence of the merging time-scale on projected separation and mass ratio, according to the prescription of (Jiang, Jing, \& Han 2014).

\section{Where are the untouched massive relic galaxies in the nearby Universe?}

The following question we want to address is whether there is any single massive nearby galaxy in our vicinity such it has remained unevolved since its early formation $\left(\mathrm{z}_{f} \gtrsim 2\right)$. 


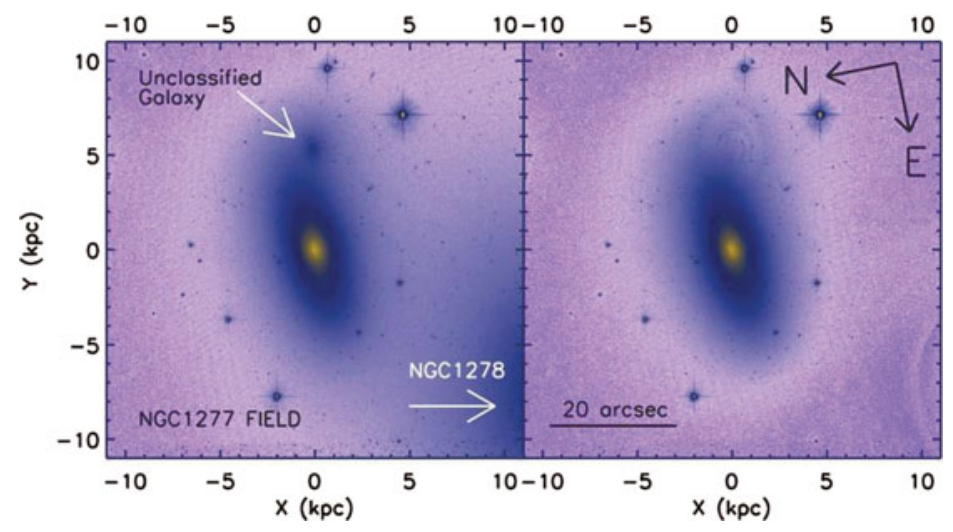

Figure 4. Neighborhood of the massive relic galaxy NGC 1277 as seen by the HST F625W filter. The left panel shows the two closest galaxies whose light contaminate NGC 1277. The right panel shows NGC 1277 after the subtraction of the contaminant light. The results indicates that NGC 1277 is rather symmetric with no distortions or bright tidal streams surrounding it.

A recent work (Quilis \& Trujillo 2013), using the Millenium simulation, suggests that 1 every 1000 massive $\left(M_{\star}>10^{11} \mathrm{M}_{\odot}\right)$ galaxies in the present-day universe has accreted less than $10 \%$ of its total stellar mass since $z=2$. Taking into account the number density of massive galaxies today, this is equivalent to find around one massive relic galaxy every $10^{6} \mathrm{Mpc}^{3}$ (i.e. one in every cosmic sphere of radius $\sim 63 \mathrm{Mpc}$ ).

Detailed analysis of our vicinity (Trujillo et al. 2009; Taylor et al. 2010; Poggianti et al. 2013) have failed to find any single massive galaxy with characteristics of a relic galaxy (i.e. $\mathrm{M}_{\star}>10^{11} \mathrm{M}_{\odot}, \mathrm{R}_{e}<1.5 \mathrm{kpc}$ and with stellar populations homogeneously old, age > 10 Gyr, along its whole structure). However, a very nearby object, NGC1277, not included in the catalogues previously used, presents structural features that makes it very attractive to be considered as a potential massive relic galaxy. In fact, its global properties $\left(\mathrm{M}_{\star}=1.2 \times 10^{11} \mathrm{M}_{\odot}, \mathrm{R}_{e}=1.2 \mathrm{kpc}, \sigma>330 \mathrm{~km} / \mathrm{s}\right.$; van den Bosch et al. 2012$)$ are within the expected properties of a galaxy with relic characteristics. We show in Fig. 4 the morphology of this galaxy located in the rich Perseus Cluster of galaxies at $73 \mathrm{Mpc}$. According to its regular morphology, NGC1277 does not present signs of currently (or in its immediate past) accreting external stars.

Not only the structural properties of NGC1277 are consistent with the structural properties of massive galaxies at $\mathrm{z} \sim 2$ but also its stellar populations are old along its radial structure (Trujillo et al. 2014) as expected if this object were a truly relic. In Fig. 5 we show the spectral energy distribution of the galaxy at different radial distances based on very deep spectra taken with the WHT telescope. At all radii, the Star Formation Histories (SFHs) of this galaxy is compatible with no new star formation in the last 10 Gyr.

Having identified a good candidate of a relic massive galaxy in the nearby Universe, this opens the possibility of exploring with exquisite detail the properties of the early steps of massive galaxy formation. In Fig. 6 we show the age, metallicity and $\alpha /$ Fe radial profiles of NGC1277. The large $\alpha / \mathrm{Fe}$ value, $\alpha / \mathrm{Fe}>0.3$, implies an extremely short formation time-scale: $\sim 100$ Myr. This short time interval translates into a huge star formation rate to form the bulk of NGC1277: $1000 \mathrm{M}_{\odot} / \mathrm{yr}$.

To conclude this section, the existence of NGC1277 strongly suggests that some massive galaxies have remained unaltered since $\mathrm{z} \sim 2$. This is in qualitative agreement with merging scenarios where some of these objects are expected to survive. These relic galaxies, on 

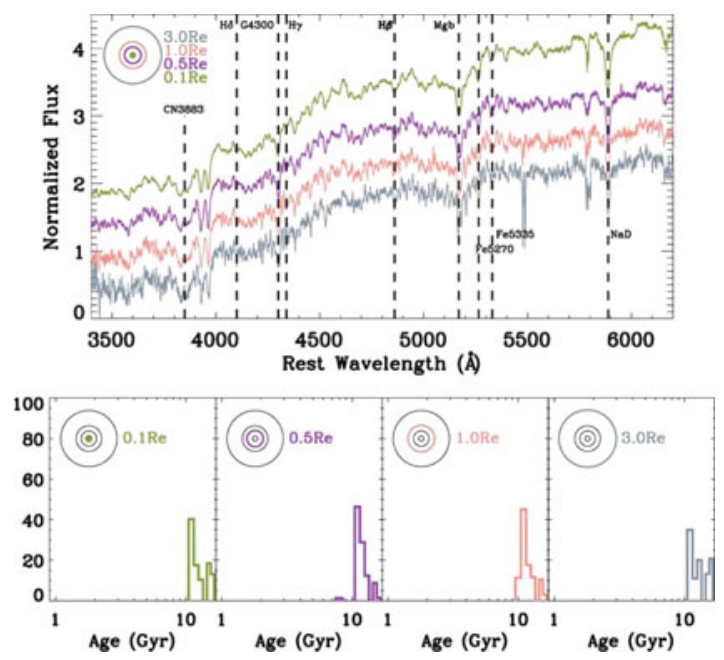

Figure 5. Spectral energy distribution of NGC1277 at different radial distances (Trujillo et al. 2014). The spectra are shown in arbitrary units, with the flux normalized per unit wavelength and shifted for clarity. The position of several relevant absorption lines are indicated with vertical lines. The bottom panels show the SFHs derived with STARLIGHT at different radial distances. This represents the fraction of mass created at each epoch.

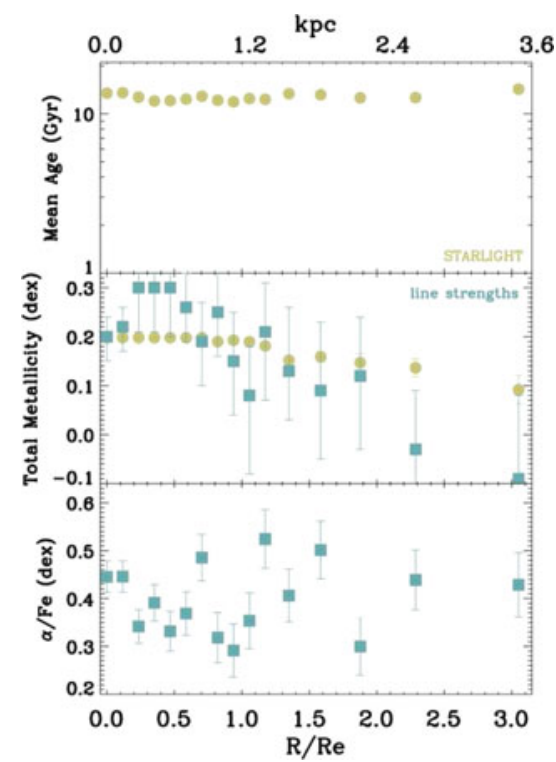

Figure 6. Age, metallicity, and $\alpha /$ Fe profiles of NGC 1277 (Trujillo et al. 2014). The figure shows the mean mass-weighted age and total metallicity derived using STARLIGHT. An independent measure of the metallicity and $\alpha / \mathrm{Fe}$ was obtained from an indices analysis. The profiles show a small change of the stellar population properties of this relic galaxy across its structure out to $3 \mathrm{R}_{e}$. It seems that the entire galaxy was formed in a unique, very fast event, which produced the high $\alpha / \mathrm{Fe}$ abundances.

the other hand, are difficult to understand in growing scenarios driven by AGN activity, as NGC1277 already hosts a very large supermassive blackhole (van den Bosch et al. 2012) and still remains compact. 


\section{Conclusions}

The merging channel mode of galaxy growth successfully passes the tests: a) of having enough number of satellites around the hosts to explain the size and mass growth, and b) it also explains the existence of unevolved massive galaxies in the nearby Universe. Future works would need to explore whether the number density of unevolved massive galaxies at all redshifts is in agreement with cosmological predictions (see e.g. Damjanov et al. 2014). In addition, it will be necessary to explore whether the outskirts of nearby massive galaxies have the same properties (age and metallicity) than the satellites found at high-z (argueably the most likely progenitors of these envelopes).

\section{Acknowledgements}

This work has been supported by the "Programa Nacional de Astronomía y Astrofísica" of the Spanish Ministry of Science and Innovation under grant AYA2010-21322-C03-02.

\section{References}

Bezanson, R., van Dokkum, P. G., Tal, T., Marchesini, D., Kriek, M., Franx, M., \& Coppi, P., 2009, ApJ, 697, 1290

Buitrago, F., Trujillo, I., Conselice, C. J., Bouwens, R. J., Dickinson, M., \& Yan, H., 2008, ApJ, 687, L61

Bundy, K., Fukugita, M., Ellis, R. S., Targett, T. A., Belli, S., \& Kodama, T., 2009, ApJ, 697, 1369

Ciotti, L. \& van Albada, T. S., 2001, ApJ, 552, L13

Daddi, E., et al., 2005, ApJ, 626, 680

Damjanov, I., Hwang, H. S., Geller, M. J., \& Chilingarian, I., 2014, ApJ, 793, 39

Fan, L., Lapi, A., De Zotti, G., \& Danese, L., 2008, ApJ, 689, L101

Ferreras, I., et al., 2014, MNRAS, 444, 906

Hopkins, P. F., Bundy, K., Murray, N., Quataert, E., Lauer, T. R., \& Ma, C.-P., 2009, MNRAS, 398,898

Jiang, C. Y., Jing, Y. P., \& Han, J., 2014, ApJ, 790, 7

Kaviraj, S., Peirani, S., Khochfar, S., Silk, J., \& Kay, S., 2009, MNRAS, 394, 1713

Khochfar, S. \& Burkert, A., 2006, A\&A, 445, 403

López-Sanjuan, C., et al., 2012, A\&̇A, 548, A7

Mármol-Queraltó, E., Trujillo, I., Pérez-González, P. G., Varela, J., \& Barro, G., 2012, MNRAS, 422,2187

Naab, T., Johansson, P. H., \& Ostriker, J. P., 2009, ApJ, 699, L178

Oser, L., Ostriker, J. P., Naab, T., Johansson, P. H., \& Burkert, A., 2010, ApJ, 725, 2312

Poggianti, B. M., et al., 2013, ApJ, 762, 77

Quilis, V. \& Trujillo, I., 2013, ApJ, 773, L8

Ragone-Figueroa, C. \& Granato, G. L., 2011, MNRAS, 414, 3690

Ruiz, P., Trujillo, I., \& Mármol-Queraltó, E., 2014, MNRAS, 442, 347

Taylor, E. N., Franx, M., Glazebrook, K., Brinchmann, J., van der Wel, A., \& van Dokkum, P. G., 2010, ApJ, 720, 723

Trujillo, I., et al., 2006, MNRAS, 373, L36

Trujillo, I., Conselice, C. J., Bundy, K., Cooper, M. C., Eisenhardt, P., \& Ellis, R. S., 2007, MNRAS, 382, 109

Trujillo, I., Cenarro, A. J., de Lorenzo-Cáceres, A., Vazdekis, A., de la Rosa, I. G., \& Cava, A., 2009, ApJ, 692, L118

Trujillo, I., Ferreras, I., \& de La Rosa, I. G., 2011, MNRAS, 415, 3903

Trujillo, I., Ferré-Mateu, A., Balcells, M., Vazdekis, A., \& Sánchez-Blázquez, P., 2014, ApJ, 780, L20

van den Bosch, R. C. E., Gebhardt, K., Gültekin, K., van de Ven, G., van der Wel, A., \& Walsh, J. L., 2012, Natur, 491, 729

van Dokkum, P. G., et al., 2010, ApJ, 709, 1018 